\title{
Development and Applications of the Minnesota Braille Skills Inventory (MBSI)
}

\author{
Michael N. Sharpe \\ University of Minnesota, United States of America
}

\begin{abstract}
The Minnesota Braille Skills Inventory (MBSI) is a standardized, norm-referenced assessment instrument which can be used to assess the braille proficiency of students 6-19 years of age. Consisting of a Literary Skills Cluster and an Advanced Skills Cluster, the MBSI can be used as a reporting tool to inform educators and parents of student progress based on normative information. Because there is also a formative component built into the MBSI, it can be used for the identification and monitoring of specific instructional goals for a student.
\end{abstract}

\section{Introduction}

Sooner or later, one of the most critical questions a teacher must ask is whether an instructional intervention has been effective in helping a student to learn. Irrespective of the subject matter, teachers have a professional obligation to find out if they have "made a difference" with students and if meaningful learning has occurred. Simply providing instruction without making some attempt to obtain feedback about intended outcomes is not just a sign of ineffective instruction; it is also unacceptable when designing effective educational plans for students with disabilities. And so it is with those who instruct students who are blind and visually impaired. Teachers who provide instruction to students with visual impairments must be prepared to offer multi-disciplinary teams, particularly the parents of children who are blind or visually impaired, with documentation that monitors student progress and supports instructional decisions. Nowhere can this point be emphasized more strongly than in the case of braille instruction. For many students, braille instruction will be the gateway of acquiring the skills that will help them to become literate and allow them to fully participate in society.

Much of the impetus to assess a student's performance in learning braille has come from an assortment of state and federal legislative initiatives passed over the last decade within the United States. Many of these efforts have been aimed at ensuring that braille remains a viable option for attaining literacy for students who are blind and visually impaired. One feature present in nearly all of these efforts is the requirement that a "comprehensive reading and writing assessment" be administered to ascertain the student's level of functioning in these two basic skill areas. However, aside from a few informal measures, teachers of the blind and visually impaired have not had access to braille reading and writing instruments that are standardized and contain normative information reflecting performance characteristics of blind and visually impaired students within the United States.

In addition to state and federal laws requiring that braille reading and writing assessments be administered to determine instructional mode, increasing emphasis is being placed on the development of measurable goals and objectives in the development of Individual Educational Plans (IEP) for students with disabilities. No longer is it satisfactory for a teacher to base instructional decisions or report student progress entirely on subjective perceptions only. Rather, the trend is toward decision making that makes use of performance-based information.

The Minnesota Braille Skills Inventory (MBSI) is designed to give teachers and parents performancebased information in the areas of braille reading and writing. The MBSI can be used by teachers to collect data about a student's level of proficiency in braille reading and writing, much like other measures of academic performance (e.g., reading recognition, comprehension, mathematics).

Similar to a norm-referenced measure of achievement, the MBSI provides teachers with scaled scores that can be used to monitor student progress over time and even make comparisons with other types of tests to facilitate instructional decisions. Also, the MBSI is standardized, meaning that it is administered the same way each time by a prescribed set of rules. Standardized test administration helps to ensure that observed differences in assessment results do not occur simply because the conditions or process used for administration had changed from one point of time to another.

Unlike many standardized, norm-referenced achievement tests, the MBSI can be used as a tool to 
develop instructional goals and objectives and to monitor performance over time. In this regard, it serves as a "criterion-referenced," or formative evaluation instrument, to identify specific instructional goals. This assessment flexibility has much to do with the nature of the assessment itself; on the MBSI, it is intended that students master as many braille symbols as possible since it is generally assumed that greater knowledge will transform itself into higher levels of literacy and hence, functionality. While there can be no guarantee that knowledge of braille symbols will ensure literacy, the opposite assumption-that braille literacy can be attained without knowledge of symbol mastery-is impossible. While other types of educational assessments are designed to "predict" future performance in particular educational domains (e.g., "reading comprehension"), the "bottom-line" focus of the MBSI is on content (i.e., code and symbol) mastery.

\section{Uses of the MBSI}

The MBSI is an assessment instrument that can be used to assess braille reading and writing skills. It addresses all the braille codes and most other symbols that students might encounter in the course of their education. It is not expected that all, or even most, students would show proficiency in all braille codes. Each student will show a different level of proficiency depending on such factors as: interest, ability, academic experience, and use of non-braille information sources.

The MBSI was designed to: (1) provide comparative information about a student's progress relative to a norm group of same-age peers who are blind or visually impaired, and (2) monitor student progress in the instructional process. Because it serves as a means of documenting student performance in these two areas, it provides a record of student performance that can be used by teachers to:

- Identify student strengths and needs in reading and writing braille.

- Develop a profile of student learning for making instructional decisions.

- Communicate objective information about a student's performance to parents and other educators and professionals.

- Obtain ongoing assessment information for a periodic review of student progress.

- Establish a consistent method of documenting student progress.

- Demonstrate accountability and support instructional decision making.

The MBSI must be used responsibly-never as the sole source on which programmatic decisions are based. Because of the high level of individual differences present in the population of students identified with visual impairments, assessment tools are best used as supplementary sources of information that contribute to the decision-making process of multidisciplinary teams.

The MBSI is designed to streamline the method of evaluating a student's knowledge of the braille code. It allows the teacher flexibility in determining which sections and subscales should be administered. Because the focus of the assessment is on content, one cluster of the MBSI can be administered as often as necessary. For example, it may be used to gather data before Individual Educational Planning (IEP) planning meetings and periodic review conferences, or it may be used even more frequently for checking a beginning braille reader's progress. This design versatility allows an experienced teacher to use the MBSI in different ways.

Although offering a wide range of uses to teachers, the MBSI is not intended to be a measure to determine learning mode or to decide how or when braille is to be taught. Other methods and tools have been developed to assist in this decision-making process. Nor does the MBSI posit itself as a braille "curriculum." Rather, it represents essential skills that would reflect a literate reader of braille. While an indepth level of comprehension and application skills are not directly assessed on the MBSI, it is unlikely that one would be able to demonstrate any degree of braille literacy without having first mastered many of the skills included on the MBSI.

\section{Organization of the MBSI}

The MBSI is organized by two major clusters: (1) the Literacy Skills Cluster and the Advanced Skills Cluster. Under each cluster are various subscales (see Table 1: Clusters and Subscales of the MBSI). The Literacy Skills Cluster allows teachers to assess student performance relative to same-age peers (i.e., normative information). The Advanced Skills Cluster is not norm-referenced; it is a measure of mastery of symbols beyond basic braille and Nemeth. In this case, mastery criteria are set by the teacher where student performance is self-referenced, rather than compared to a norm group. Both clusters of the MBSI include various subscales. Subscales contain items of similar content in order to assess student performance in specific domain areas (e.g., alphanumeric, whole words, music).

The Literacy Skills Cluster serves a very important assessment function on the MBSI as it allows for the grouping, or aggregation, of all seven subtests to obtain one comprehensive estimate of performance. Referred to as a "composite score," this clustering of all subscales into one general estimate of performance strengthens overall reliability with regard to making an accurate assessment of a student's overall level of proficiency. The Literacy Skills 
Cluster is the only composite score that can be obtained on the MBSI.

The Advanced Skills Cluster of the MBSI represents intermediate and "specialized" symbols that in many cases are more suitable for middle and second-

Table 1: Clusters and subscales of the MBSI

\section{Literacy Skills Cluster}

The Literacy Skills Cluster consists of seven subscales that represent fundamental skills necessary for academic functioning. The primary purpose of the Literacy Skills Cluster is to obtain normative information about a student's performance. Subscales of the Literacy Skills Cluster:

1. Alphanumeric

2. Whole Words

3. Short Form Words

4. Part Words

5. Two Cell Contractions

6. Punctuation and Composition

7. Basic Nemeth

\section{Advanced Skills Cluster}

The Advanced Skills Cluster consists of three subscales that contain braille symbols necessary for advanced instruction or specialized learning. The primary purpose of the Advanced Skills Cluster is to obtain criterion-referenced information about a student's performance. Subscales of the Advanced Skills Cluster:

8. Advanced Nemeth

9. Computer

10. Music

ary-level students who are more likely to demonstrate a need to learn more advanced symbols in the areas of mathematics, computer related text, and music. The results of the Advanced Skills Cluster have neither been designed nor are intended to be compared to a norm group. Rather, their purpose is to help teachers set instructional objectives and facilitate the monitoring of student performance.

\subsection{The Literary Skills Cluster}

Subscale items within the Literacy Skills Cluster represent the building blocks fundamental to a student's ability to achieve braille literacy. As primary skills, the symbols of this cluster area are often introduced at an early age, beginning with the alphabet and growing with increasing complexity to intermediate level reading and writing skills. As such, the Literacy Skills Cluster represents the "core knowledge" of braille literacy, consisting of basic literacy and Nemeth symbols. By demonstrating a thorough mastery of the symbols contained in the Literacy Skills Cluster, it can be concluded that a student will have achieved many of the requisite reading and writing skills necessary to engage in self-directed learning activities. This includes the ability to read textbooks, newspapers, magazines and other learning materials which are available in braille. Similarly, it includes the ability to communicate using written language skills so that it can be read by other readers of braille, or transcribed into print so that it can be read by non-braille readers.

The Literacy Skills Cluster consists of both reading and writing activities in which normative data are available for students ages 6 to 19. These norms allow teachers to obtain information about a student's MBSI performance relative to a group of same-age peers. Teachers are able to utilize standard scores and percentiles to determine whether a student's performance is "above" or "below" age expectations using the normative group as a frame of reference.

To facilitate the assessment process, many of the items included on the various subscales of the Literacy Skills Cluster have been placed in a sequence that generally approximates the difficulty level in which students learn to read and write braille. However, this does not necessarily imply that braille reading and writing should be taught according to the organization of the MBSI. Once again, decisions of this nature are best left to teacher judgment with input from the multidisciplinary team.

\subsection{Description of the Advanced Skills Cluster}

The Advanced Skills Cluster of the MBSI serves as a criterion-referenced measure which can be used by teachers to identify learning objectives, set standards of "mastery," and monitor student performance. As suggested by its name, the Advanced Skills Cluster contains braille symbols that are generally learned in specialized types of reading and writing applications (e.g., computer, music) or to master skills of increasing complexity (e.g., Advanced Nemeth). Subscales and general contents within each are shown below.

The Advanced Skills Cluster of the MBSI is based on a criterion-referenced approach to assessment-the student's performance is not assessed relative to other peers who use braille; rather, assessment is based on referencing student performance in terms of a criterion.

Performance criteria will vary from one student to the next, depending largely on the teacher judgment of student needs. As such, the Advanced Skills Cluster can operate as a checklist of key skills that need to be mastered in order for the student to achieve a specific instructional goal. For example, if one of the student's learning objectives is to learn how to read and write music, the teacher needs to determine: (1) the student's present skill level in interpreting braille 
musical symbols, (2) the reading and writing skills needed to achieve the intended objective, and (3) the criteria that will be used to assess whether mastery has been achieved.

\section{Item Development}

Work began in the development of the MBSI in the early 1990's, when staff from the Minnesota Resource Center and the Minnesota State Vision Network formed a joint committee to plan and develop a reading and writing skills inventory that would meet the intent of Minnesota's newly created braille law. One of the first of its kind in the U.S., this legislation mandated that a "comprehensive reading and writing assessment" be provided for students with visual impairments. The planning committee consisted of Minnesota teachers licensed in the area of vision impairment who represented a variety of educational settings: itinerant, residential, urban, suburban, and rural.

The committee met regularly over the course of two years to: (1) develop the conceptual framework of the instrument, (2) establish the central purposes of the assessment, and (3) identify the range of content to be included in the assessment. Throughout the developmental stages of this effort, one of the key issues addressed was whether braille skills should be assessed in context or isolation. While acknowledging the importance of learning skills in context, it was concluded that such an assessment would be prohibitive given its wide range of item content. However, as item development progressed, it also became clear that some braille symbols needed to be presented in context to ensure that students were given the opportunity to make discrete and unambiguous responses to specific types of items.

Once the general design had been completed, work began on the development of items. This task started with an extensive review of the literature and the use of group consensus processes to identify and select a comprehensive pool of items that students need to read and write braille. To facilitate this process, items were assigned to "sections" based on their logical placement from the range of braille codes available; these included: Literary, Basic Nemeth (mathematics), Advanced Nemeth, Computer, and Music. The rationale for including each major section is described in the following section.

\subsection{Literary Code}

The Literary Code comprises the largest part of a student's braille literacy program, and it is where most of the research and curriculum information is devoted. Because of the wide range of content in Literary Code, it was necessary to create subscales of common content (e.g., alphanumeric) to facilitate the assessment process. Also, punctuation and composition symbols commonly found in text were included in this section.

\subsection{Basic and Advanced Nemeth}

Nemeth symbols are those that students need for mathematics instruction. Several curricula were reviewed and mathematics instructors were consulted for input in the item selection process. Because of the many braille symbols used in advanced math studies, only the most frequently used were included. One problem encountered in developing items for this portion of the MBSI was that not all Nemeth braille symbols have an exact correspondence with printed math signs. As such, it was necessary to develop items in which math problems were presented in context.

\subsection{Music}

Despite the few resources available for teaching Music code, this area was considered to be essential to ensure that all key aspects of a modern school curriculum would be included on the assessment. To determine item content, technical assistance was sought out from experts in the field of music, including braillist, musicians, and college-level music majors who read and write in braille.

\subsection{Computer}

Computer items were drawn from the computer braille code and reflect the range of skills necessary to demonstrate competence with various aspects of technology. Consultants who assisted committee members with this area included a braillist, a specialist at a technology resource center for individuals who are blind and visually impaired, and a computer specialist. All technology specialists were individuals who personally use braille.

Once the instrument was developed as the MBSI Field Test Edition, it was field-tested by teachers of the visually impaired in Minnesota. Field testing was conducted by videotaping the administration of the MBSI Field Test Edition by a single examiner to five students of varying skills and abilities. Throughout the administration, the examiner scored each response and took care not to reveal scoring preferences (i.e., whether a student passed, or failed to pass, a given item). Once the videotaped administration of the assessment was completed, nine teachers independently viewed the tapes and made judgments about how each individual would score the student. Altogether, the teacher raters scored eight different MBSI Field Test Edition protocols. Five of the protocols were developed as reading samples and three as writing samples. The data obtained from this activity were used to obtain preliminary information about 
the interrater reliability of the MBSI and to finalize the overall format of the instrument.

With interrater reliability coefficients being available for every combination of nine raters (i.e., rater 1 with rater 2 ; rater 1 with rater 3 ), a total of 36 coefficients were obtained. In an analysis of these various combinations, interrater reliability coefficients for the reading samples were found to range from .83 to 1.00 , while writing sample coefficients ranged from .93 to 1.00 . Mean interrater reliability coefficients for reading samples ranged from .94 to 1.00 , while writing samples ranged from .91 to .99 . In sum, these preliminary interrater reliabilities revealed strong relationships among ratings, often approximating "perfect" reliability. However, it is important to note that high reliability does not mean that different raters gave each protocol the same scores-although this phenomenon was frequently observed. High interrater reliability only means that different raters' scores ranked people in the same relative order. As such, any two raters may produce slightly different scores, but if their scores consistently rank people in the same relative order, the reliability can be perfect.

\section{Standardization and Technical Procedures}

\subsection{Norming Procedures}

Once the instrument format was finalized, norming procedures ensued. These activities began in the 1996-97 academic year by identifying and recruiting professionals in the field of blindness and visual impairments to participate. A database of names obtained from membership lists of professional organizations was used to recruit participants. From these lists, over 1,000 recruitment letters were mailed to professionals in the field. As a result of the mailing solicitation, 333 professionals agreed to either participate in norming efforts or provide teaching strategies for effective braille instruction. The latter was an activity related to a separate study and did not require involvement in norming activities.

As a result of this recruitment effort, data was obtained from 354 students across the United States. Thus, these students comprised the MBSI norm group. Approximately $82 \%$ were identified as Caucasian, varying only slightly from the U. S. population of approximately $80 \%$. African American and Hispanic students were represented adequately relative to the overall population. With regard to gender, $49 \%$ of the sample of students in the norm group were male, while $51 \%$ were female. When examining the demographic information of the norm group, it was observed that approximately $38 \%$ of the students in the norm group were identified with another disability other than a visual impairment. More details about the sample and norming process can be found in the MBSI Examiner's Manual (Sharpe \& McGrew, 2013) [1].

\subsection{Item Analysis}

Within each subscale (e.g., Alphanumeric, Basic Nemeth), all items were analyzed to determine whether they met the requirements of the oneparameter Rasch IRT model (Rasch, 1960 [2]; Wright, 1968 [3]; Wright \& Linacre, 1991[4]; Wright \& Stone, 1979 [5]). These analyses were completed with the BIGSTEPS (Version 2.1) Rasch analysis program (Wright \& Linacre, 1991[4]). All Rasch item difficulty and person ability values for each subscale were converted to the $W$ scale, a special transformation of the Rasch difficulty and log ability scales (Woodcock \& Dahl, 1971[6]) that has been used in a variety of psychoeducational assessment instruments such as the Woodcock-Johnson Psycho-Educational Battery-Revised (WJ-R) (Woodcock \& Johnson, 1989 [7]) and the (Woodcock-McGrew-Werder Mini-Battery of Achievement (MBA) (Woodcock, McGrew, \& Werder, 1994 [8]).

There are distinct advantages to having scores on the equal interval $W$ scale (e.g., for measuring change and conducting statistical analyses) that are absent when using raw scores (see Woodcock, 1978 [9]) for a detailed treatment of these issues). A more detailed discussion of the advantages of using the Rasch model and the $W$ scale in the development of tests can be found in McGrew, Werder, and Woodcock (1991 [10]) and Woodcock (1978 [9]). Item difficulty information conveyed by item $W$ scores was used to determine the final ordering of items with each subscale. That is, the items within each subscale on the Literacy Skills Cluster were ordered from the least to the most difficult according to the item $W$ difficulty values.

In addition, the Rasch scaling procedures produced a Raw-to- $W$ Score scoring table for each subscale. Each of these scoring tables showed the 1-to-1 correspondence between all possible raw scores for each subscale and the respective $W$ person ability scores. That is, the scoring table converted subscale raw scores to the equal interval $W$ score person ability metric, a metric with more desirable measurement characteristics for developing test norms and derived scores. The final scoring tables for each subscale were used to assign each norming subject a $W$ ability score for each subscale, thus producing equal interval measures for each subject for each subscale that were then used in the calculation of the MBSI norms and derived scores.

\subsection{Calculation of Derived Scores and Norms}

For each subscale on the Literacy Skills Cluster, a variety of scores can be obtained. These include total raw scores, $W$ ability scores, age-equivalents, stand- 
ard scores $(\mathrm{M}=100 ; \mathrm{SD}=15)$ and percentile ranks. However, only standard scores and percentile ranks are used to report results when administering the MBSI. Age-equivalent scores and $W$ ability scores are used only to report results of the norming process and illustrate overall performance differences between age groups. The only exception to this rule is when $W$ ability scores are used to compute the composite of the Literacy Cluster. Age-equivalent scores for each scale were obtained by first grouping the norming subjects into 14 successive age-based subsamples. The mean $W$ score for each subscale at each of the age levels was calculated and plotted by mean age. The mean $W$ scores were "smoothed" by a polynomial curve-fitting and equation discovery software program (Table Curve 2D; Jandel Scientific, 1996 [11]) to provide the "best fit" relative to the means. The age-equivalent scores produced by the final smoothed curves were then recorded in the appropriate tables. An example of the curve fitting results for mean $W$ scores for the Short Form Words subscale is presented in Figure 1: Mean $W$ Scores.

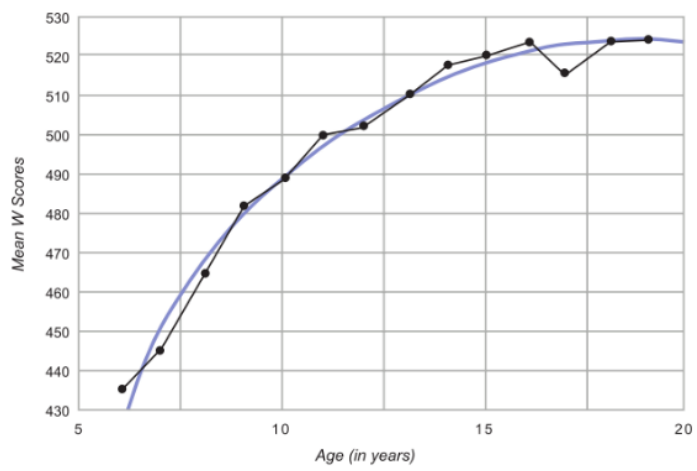

Figure 1: Mean $W$ scores

Inspection of Figure 1 reveals a clear developmental progression in the mean scores with age, a finding that indicates that the Short Form Words subscale is measuring an ability or trait that is developmental in nature. It can be seen in Figure 1 that the sample-based mean $W$ scores (the values represented by the circles), although showing a monotonically increasing developmental trend, did not display a perfectly smooth pattern of growth. This is typical for plots of sample-based parameter estimates for developmentally based human ability traits and reflects the effects of sampling error. The smoothed curve presented in the table represents the best "fit" to the data points and represents the best estimate of the population-based developmental growth pattern in the mean $W$ scores. The data for all MBSI subscales showed the same developmental trends as in Figure 1. The derivation of the age-equivalents for the Short Form Words subscale was obtained by "reading" from the smoothed curve the age (in years and months on the x-axis) that was associated with the corresponding $W$ ability score (the y-axis). That is, each point along the smoothed norm curve represents a unique value that is represented by a particular $\mathrm{x} / \mathrm{y}$ intersection. These points represent the typical or average performance (in $W$ scores) for each particular age. These values were used as the ageequivalents for the Short Form Words subscale. To reduce the complexity of the MBSI scoring, the Rasch scoring table of the Short Form Words subscale, for example, was then used to convert the $\mathrm{W}$ scores back to the raw score scale. This transformation allowed for the provision of direct raw score to age-equivalent scoring tables. The desirable equalinterval measurement characteristics of the $W$ scale were used to develop the age-equivalents and are the foundation for all the MBSI norm tables, but the $W$ score metric is "transparent" to the user.

\section{Summary Statistics}

Although not shown in this article, the MBSI Examiner's Manual (Sharpe \& McGrew, 2013 [1]) contains detailed tables such as the Means and Standard Deviations-Reading and Means and Standard Deviations-Writing tables to summarize the descriptive data for the MBSI subscales for all age groups. The manual also contains table information about means and standard deviations expressed in $W$ scale units. For all subscales, the summary statistics show a systematic developmental increase in average scores from younger to older age groups. The finding of a monotonically increasing developmental trend is consistent with that shown by most standardized norm-referenced instruments that measure human abilities/traits. (See summary statistics and developmental growth curves for the Woodcock-Johnson Psychoeducational Battery-Revised; McGrew et al., 1991 [10]). Developmental increases in test scores is frequently considered to be one indicator of the validity of a measurement scale

\subsection{Validity}

Validity information for the MBSI instruments is currently available in the form of content validity, analysis of changes in scores as a function of age, and the intercorrelation of scores from the subscales. External validation in the form of concurrent or predictive correlations with measures of similar or different constructs is currently not available.

\subsection{Content Validity}

Content validity refers to the extent to which the items in a test represent the domain of ability that it is designed to measure. Items included in the MBSI 
were carefully selected to represent the range of braille symbols that are needed for academic achievement. An extensive process of item development was employed to ensure that content would indeed reflect those skills deemed most important by teachers. At each point in this process, items underwent numerous edits and revisions in response to the feedback, consultation, and critiques provided by both braille teacher experts and braille users. Certified braille transcribers were also consulted regarding unique aspects of the braille code as well as general education teachers who provide advice in such specific areas as mathematics and computer related text. Given these efforts, the content included in the MBSI can be considered a valid measure of student performance in the area of braille instruction.

\subsection{Age-Related Analyses}

Analysis of the change in MBSI scores as a function of age can be found in Figure 1 and table included in the MBSI Examiner's Manual (Sharpe \& McGrew, 2013 [1]) As previously noted, Figure 1 revealed a distinct systematic developmental increase in the average Short Form Words subscale scores (the same trend was present for all MBSI subscales) as a function of increasing age. This systematic agerelated trend provides support for the MBSI subscales, as most measures of academic abilities (i.e., reading, writing, and math) are known to increase developmentally with age. Age-related changes in measures of developmental constructs is often considered as one form of construct validity for the measures.

\section{Administration and Scoring Procedures}

\subsection{Administration of the MBSI}

The Minnesota Braille Skills Inventory (MBSI) must be administered by a teacher who is licensed in teaching students who are blind or visually impaired. To ensure accurate results, teachers must become thoroughly familiar with administration content and faithfully follow instructions before administering the assessment.

In order to administer the MBSI, the teacher will need the following information, response devices and materials:

- A series of administration prompts which are used by the teacher to administer the MBSI subscales in a standardized manner.

- A MBSI Student Record Form which is used by the teacher to record student responses for both reading and writing subscales.
- A MBSI Student Response Book, a brailed version of the assessment items which is used by the student to respond to the reading subscales of the MBSI.

- A braille writing device (e.g., a Perkins or other AT device) which is used by the student to respond to the writing subscales of the MBSI.

The MBSI requires the student to perform two types of tasks: reading and writing. The MBSI Student Response Book is needed by the student to perform reading tasks, while a braille writing device must be used by the student to complete writing tasks. In each case, the teacher will use the MBSI Student Record Form to record responses and the MBSI Examiner's Manual to reference tables of normative data for the Literacy Skills Cluster.

There are no specific rules regarding the order in which the MBSI is administered, although most will find it convenient to administer the reading tasks first, followed by the writing assessment activities. Similarly, it does not make a difference whether one chooses to administer only the Literacy Skills Cluster or the Advanced Skills Cluster, or both. These decisions should be dictated by the individualized learning needs of the student.

Logically, administration time will vary based on the student's age and the number of subscales administered. In cases where both reading and writing tasks of the Literacy Skills Cluster and Advanced Skills Cluster will be administered, the teacher may wish to use more than one session. However, even though administration can be conducted for more than one session, it is urged that the span of time to complete the entire assessment should not exceed two weeks.

There is no time limit for the MBSI; examiners should use their own judgment about how much time a student should spend on any one item. However, there will be times when the examiner must exercise caution in order to avoid having the student become too frustrated or discouraged. To help avoid this situation, a "discontinue rule" has been established for the Literacy Skills Cluster to help move the assessment process along without having to overtax efforts made on behalf of students. A discontinue rule does not exist for the Advanced Skills Cluster of the MBSI since there is a "set" order of the items. Rather, teachers are expected to select items based on student needs and only administer those items.

A number of items (e.g., math, punctuation, and money symbols) within the MBSI are presented in "context" so that students can be provided with realistic examples. These items are included MBSI Student Response Book so that students have the opportunity to identify the symbol in a context that more closely approximates what is actually experienced in an instructional setting. First, the student can examine the symbol, then use the contextual elements 
within the example to provide a response to a question.

To maintain consistency in the way the MBSI is administered, a series of verbal prompts have been prepared for reading and writing tasks in. These represent a standard set of instructions that the teacher examiner can use to communicate what is expected of the student.

\subsection{Scoring of the MBSI}

The MBSI scoring procedure consists of a series of items in which the teacher assigns a "1" if the student can demonstrate mastery of the item, or a " 0 " if the student does not know the item. Administering items from the MBSI requires a direct response from the student.

To obtain scores which can be used to interpret norm-referenced performance, one must first determine how many items were "passed," or scored as a "1" within each subscale. These constitute the "raw scores" and will be used to obtain other types of scores that can be used to describe a student's performance in the Literacy Skills Cluster. Subscale raw scores can be transformed into standard scores and percentiles for purposes of interpretation. While $W$ ability scores are used in the process to derive the Literacy Cluster Composite, which is a summary of literacy subscales, this composite score is still expressed as a standard score. Descriptions of standard score and percentile rank follow:

Standard Score - A derived score which is based on an average or "mean" score and can be described in terms of its range of variability-the standard deviation. On the MBSI, a statistical average is set at 100 with a standard deviation of 15 . This mean and standard deviation is commonly used in most major norm-referenced tests of ability and achievement.

Percentile Rank - Familiar to most people, the percentile rank represents a "point" on a scale for a set of scores. As such, a specified proportion of cases in a distribution can fall at or below a given percentile rank. On the MBSI, a standard score of 100 represents the $50 \%$ percentile rank-the point in which half of the sample scored above or below.

\section{How the MBSI Can Be Used}

The results of the Minnesota Braille Skills Inventory (MBSI) offers information to the teacher which can be used in various ways to enrich the braille instruction of students. For example, as a reporting tool, the MBSI can be used to inform parents and others of the student's progress based on normative information. Also, it can be used for the identification and monitoring of specific instructional goals for the student. Use of the MBSI will depend upon the purposes of the assessment. Using results obtained from each cluster area is discussed in the following section.

\subsection{Literacy Skills Cluster}

The Literacy Skills Cluster of the MBSI provides normative information about the student. As such, teachers will use the results to see how the student is progressing relative to same-age students who are being given braille instruction. Looking at a student's performance in a normative context helps teachers to address the important question, "Is the student learning to read and write braille at a rate commensurate with peers?" The Literacy Skills Cluster helps to gauge student performance that not only permits making comparisons from one test administration to another, but also with a variety of other tests and measurements as well.

Interpretation of the Literacy Skills Cluster is based on a standard score mean of 100 and a standard deviation of 15 . As such, a standard score of 100 on any subscale, including the Literacy Cluster Composite, represents the 50th percentile. The standard deviation helps to further define the range of performance that can help one to determine whether a particular score is "high" or "low" relative to the mean. Like many other commonly used assessments, one standard deviation above or below the mean generally constitutes the "average" range of performance. Thus, an obtained standard score of 84 or below would reflect "below average" performance.

Despite the ease with which MBSI scores can be interpreted, users of the assessment should refrain from reporting results without stressing that all assessment results, irrespective of type (e.g., cognitive ability, achievement) contain some degree of measurement error. More precisely, the obtained standard scores are really representative of some "midpoint" of range of scores that often vary due to such factors as age, item difficulty, etc. Simply put, the obtained score for any MBSI subscale or Literacy Skills Cluster is only a point-in-time estimate of performance and should be interpreted as such - not as a "fixed" result not subject to change. While infrequent, wide variations in standard score results may sometimes occur due to developmental changes in the student or as different types of instructional interventions are implemented.

In addition to treating obtained standard scores and percentiles as estimates of a student's performance, users of the assessment, particularly experienced teachers, should also be careful not to abandon their clinical impressions of the student. Knowledge gained from diagnostic teaching activities, observations, and just by talking with the student also need to be factored into the "mix" to enrich interpretation of the MBSI. For example, having an awareness of 
the student's learning style, level of motivation, and overall readiness to learn braille symbols will add a broader dimension to the interpretation and will help parents to gain a better understanding of the results and the instructional implications for the future. While the Literacy Skills Cluster of the MBSI has been designed as an empirically based instrument that yields valid and reliable results, its usefulness to teachers is largely as a tool that provides meaningful feedback in instructional settings.

\subsection{Advanced Skills Cluster}

Interpretation of the Advanced Skills Cluster is generally quite straightforward - all one needs to do is to scan each item to obtain an impression of what braille symbols the student does and does not know. By doing so, the teacher will obtain an idea of the student's current skill level and determine whether any gaps exist in relation to instructional expectations. As indicated previously, much of this process will depend on the information collected prior to administering the MBSI. Also, the teacher will need to exercise clinical judgment to determine whether teaching interventions have been successful or if any changes should be made in the future.

To a large extent, the interpretation process is really one that attempts to identify discrepancies that exist between what the student "ought" to know and the student's current repertoire of skills. Naturally, the objective of instruction is to reduce the number of these discrepancies to where the student has mastered all of the braille symbols necessary to advance learning in the future. For many students, this process will be ongoing. As they move from one grade to the next, there will be a continuing need to introduce new symbols to address new learning needs. Similarly, at times it may be necessary to reintroduce symbols that were previously learned to reinforce retention. Once again, this will vary from student to student based on individual learning needs.

\section{Conclusion}

The Minnesota Braille Skills Inventory (MBSI) is a standardized, norm-referenced assessment instrument which can be used to assess the braille proficiency of students 6-19 years of age. Consisting of a Literary Skills Cluster and an Advanced Skills Cluster, the MBSI can be used as a reporting tool to inform educators and parents of student progress based on normative information. Because there is also a formative component built into the MBSI, it can be used for the identification and monitoring of specific instructional goals for a student. In general, reliability and validity data show that the MBSI can be confidently used to assess braille proficiency of children with visual impairments. However, as with all types of assessment instruments, the MBSI cannot be used as the sole source of assessing proficiency. Clearly, this task is one in which multiple sources of evidence must be used - and it is the convergence of the evidence, along with clinical insights - that are critical to the assessment process. The MBSI represents one instrument that has demonstrated proven value in the diagnostic and educational planning process for students with visual impairments.

\section{References}

[1] Sharpe, M .N. \& McGrew, K. S. (2013). MBSI examiner's manual. Sigma Associates, Inc.

[2] J. Rasch, G. (1960). Probabilistic models for some intelligence and attainment tests. Copenhagen, Denmark: Danish Institute for Educational Research.

[3] Wright, B.D. (1968). Sample-free test calibration and person measurement. Proceedings of the 1967 Invitational Conference on Testing Problems (pp. 85-101). Princeton, NJ: Educational Testing Service.

[4] Wright, B.D. \& Linacre, J.M. (1991). BIGSTEPS: Rasch-model computer program. Chicago: MESA Press.

[5] Wright, B.D. \& Stone, M.H. (1979). Best test design. Chicago: MESA Press.

[6] Woodcock, R.W. \& Dahl, M. N. (1971). A common scale for the measurement of person ability and test item difficulty (AGS Paper No. 10). Circle Pines, MN: American Guidance Service.

[7] Woodcock, R.W. \& Johnson, M.B. (1989). WoodcockJohnson psycho-educational battery revised. Chicago: Riverside Publishing.

[8] Woodcock, R.W., McGrew, K.S., \& Werder, J.K. (1994). Woodcock-McGrew-Werder mini-battery of achievement. Chicago: Riverside Publishing.

[9] Woodcock, R.W. (1978). Development and standardization of the Woodcock-Johnson psycho-educational battery. Chicago: Riverside Publishing.

[10] McGrew, K. S., Werder, J. K., \& Woodcock, R. W. (1991). Woodcock-Johnson technical manual. Allen, TX: DLM Teaching Resources.

[11] Jandel Scientific (1996). Table curve 2D: automated curve fitting and equation discovery. San Rafael: CA, Author. 